\title{
RAPPRESENTAZIONE ARMONICA DEL CICLO DI ISTERESI MAGNETICA ').
}

\author{
Nota $d i$ F. PIOLA.
}

\section{Introduzione.}

La magnetizzazione delle sostanze ferromagnetichè̀, come ̀̀ noto, funzione, oltre che dello stato attuale del campo magnetico al quale il materiale è sottoposto, anche di tutti gli stati precedenti, presentando quel fenomeno che vien detto di isteresi magnetica ed al quale corrisponde dissipazione di energia. Inoltre, per uno stesso campo, pur raggiunto seguendo gli stessi stati successivi, la magnetizzazione di uno stesso materiale differisce col modo nel quale il materiale stesso è stato cimentato meccanicamente e termicamente talmentechè due campioni, inizialmente identici, vengono, dopo essere stati diversamente cimentati, a comportarsi come se appartenessero a materiali distinti.

Pur tuttavia so si prende un certo campione che abbia seguita una certa storia meccanica e termica, e si sottopone a campi magnetici variabili fra valori uguali e di segno contrario, dopo un numero sufficiente di inversioni, dopo cioè che sia stato, come ordinariamente si dice, ciclizzato, si ottengono per la magnetizzazione successioni di valori indefinitamente sovrapponentisi e l'andamento generale del fenomeno assume delle ben note caratteristiche per cui la curva che ne risulta ciclo simmetrico di isteresi magnetica - è riconoscibile a prima vista.

Ad onta della costanza nella forma generale, una rappresentazione analitica soddisfacente della magnetizzazione in fun-

') Il presente lavoro è stato pubblicato in largo sunto in a L'Elettrotecnica » del 5 novembre 1917. 
zione periodica del campe non è stata ancora data per quanto molti tentativi siano stati fatti in proposito. Si hanno, è vero, delle equazioni ${ }^{1}$ ) che rispechiano abbastanza fedelmente, sotto certe condizioni, i rami separati della curva, o loro porzioni, ma esse, pur potendo essere utili in certi casi speciali, non hanno quella forma periodica che è necessaria se vogliamo poter introdurre nei calcolı le peculiari qualità delle sostanze ferromagnetiche nel più gran numero delle applicazioni pratiche.

Le rappresentazioni che oggi vengo no impiegate non danno le particolarità più importanti del fenomeno: il più spesso, infatti, ci si accontenta di stabilire la semplice proporzionalità della magnetizzazione al campo facendo uso, come se si si trattasse di una costante, del loro rappor‘o $k$, ciò̀ di quella che vien detta la suscettività magnetica, od introducendo la permeabilità $\mu$ legata alla precedente dalla relazione:

$$
\mu=1+4 \pi k \text {. }
$$

E poichè suscettività o permeabilità - delle quali si ̀̀ persino incerti sulla definizione quando magnetizzazione $\theta$ campo non partono ambedue dallo zero - sono ben lungi dall'essere costanti, si introduce il loro valore medio, - concetto anche questo più che mai incerto.

Quando si abbia bisogno di una maggiore approssimazione - e restringendosi al caso della magnetizzazione simmetrica, che è poi quello che più interessa nelle applicazioni, - si identifica la curva rappresentante il fenomeno con una elisse della quale è ben lungi da ricordare la forma. Ciò equivale a supporre che, contrariamente a quanto si faceva nella rappresentazione precedentemente considerata, la magnetizzazione segua il campo con un ritardo costante.

Detto $x$ il campo ed $y$ la magnetizzazione, ed H, I i loro valori massimi, si pone:

$$
\begin{aligned}
& x=\mathrm{H} \operatorname{sen} \alpha=\mathrm{H} \operatorname{sen}{ }_{\mathrm{T}}^{2 \pi} t \\
& y=\mathrm{I} \operatorname{sen}(\alpha-\varphi)=\mathrm{I} \operatorname{sen} \frac{2 \pi}{\mathrm{T}}(t-\tau),
\end{aligned}
$$

1) I.ord Rayletgh: Phil. mag. v. 23, 1887, pag. 225. - Cisotti : Rend. Lincei, จ. 17, 1908, pag. 413. 
dove l'angolo a od il tempo $t$ crescono indefinitamente e dove $\varphi$ e $\tau$ rappresentano il ritardo contato rispettivamente in angolo 0.1 in tempo, secondo più conviene.

Eliminando $\alpha$ fra le due equazioni si ottiene:

$$
y^{2}-2 \frac{\mathrm{I}}{\mathrm{H}} \cos \varphi \cdot x y+\left(\frac{\mathrm{I}}{\mathrm{H}}\right)^{2} x^{2}=\mathrm{I}^{2} \operatorname{sen}^{3} \varphi
$$

che rappresenta una elisse col centro nella origine delle coordinate, inscritta nel rettangolo di vertici $\pm \mathrm{H}, \pm \mathrm{I}$ ed avente gli assi inclinati degli angoli $\psi$ e $\frac{\pi}{2}+\psi$ sull'asse delle $x$ essendo $\psi,-$ se si indicano $\operatorname{con} \theta$ e $\pi-\theta$ gli angoli formati collo stesso asse delle $x$ dalle diagonali del detto rettangolo, dato dalla relazione:

$$
\operatorname{tg} 2 \psi=\operatorname{tg} 2 \theta \cdot \cos \varphi .
$$

Il ritardo $\varphi$ è determinato stabilendo che l'area

$$
\mathrm{W}=\pi \mathrm{HI} \operatorname{sen} \varphi
$$

della elisse uguagli quella del ciclo di isteresi effettivamente percorso dal materiale che si considera. Tale area misura la energia dissipata nel fenomeno la quale si suppone nota o per determinazione sperimentale diretta o per applicazione di formuie empiriche. Il ritardo $\varphi$ cresce con la energia dissipata in guisa che, se esistono due materiali i quali collo stesso campo nnassimo acquistano la stessa magnetizzazione ma dissipano differente quantita di energia, compete $\varphi$ maggiore a quello che ne dissipa di più.

L'artificio della elisse ha reso segnalati servigi in elettrotecnica, ma nemmeno esso è sufficiente quando si vogliano studiare più da presso $\mathrm{i}$ fenomeni che con quello della isteresi magnetica sono collegati. Esso infatti, se tiene conto della energia dissipata per isteresi, trascura - ciò che, per certi riguardi, costituisce la caratteristica principale del fenomeno l'essere la magnetizzazione sempre crescente e decrescente al crescere e decrescere del campo, talmentechè coincidono i valori più elevati dell'uno con quelli dell'altra. Colla rappresentazione a mezzo della elisse, quando il campo ha raggiunto il suo valore massimo $H$, la magnetizzazione non è $\operatorname{che} I \cos \varphi$ e non di- 
venterà massima, cioè I, se non quando il campo sarà gì̀ decresciuto ad $H \cos \varphi$. Di qui ne viene che la suscettività vera ${ }^{\text {), }}$, cioè la derivata della magnetizzazione rispetto al campo - l'unica che abbia un significato fisico ben determinato è rappresentata da una funzione che muta segno, mentre in fatto si conserva sempre positiva.

La coincidenza dei valori più elevati si aveva colla rappresentazione rettilinea, ma con essa non si teneva conto della energia dissipata.

In alcuni casi si può avere la illusione che la elisse rappresenti bene il fenomeno di isteresi. Infatti, se si sperimenta creando il campo con la ordinaria corrente alternata e rivelando gli effetti sul materiale allo studio con un oscillografo senza inerzia, p. es. col tubo di Braun, colla solita disposizione - e cioè producendo le deviazioni proporzionali al campo con un circuito in serie con quello che dà il campo stesso - si osserva, quando il materiale non sia in filo o lamine abbastanza sottili, una figura risultante che somiglia tanto più ad una elisse quanto maggiori sono le dimensioni del materiale normali al campo. Ma è noto che, in questo caso, è l'effetto delle correnti di Foucault che, più o meno, maschera quello della isteresi, poichè per queste correnti ${ }^{2}$ ) la magnetizzazione viene sfasata sul campo.

Una rappresentazione analitica più soddisfacente delle precedenti è stata suggerita da Silvanus Thompson ${ }^{3}$ ). Egli, supposta, come nel caso della elisse, una delle variabili sotto forma

1) Chi scrive ha già altra volta mes:o in rilievo la opportunità di chiamare suscettività vera, invece che differenziale, la derivata della magnetizzazione rispetto al campo. Per suscettività media in un certo intervallo intenderemo la media dei valori presi dalla suscettività vera nell'intervallo: tale rapporto coincide con quello della variazione totale della magnetizzazione alla corrispondente del campo. Analogamente vien detto per la permeabilità vera e media. (Atti A: E. T., 1911, giugno. - Nuovo Cimento ", 1912, maggio, nota al paragrafo 4 ).

2) PIOLA: \& Le correnti di Foucuult nel nucleo conduttore interuo ad un solenoide percorso da corrente alternita. Rend. Lincei, I sem., 1907, p. 35.

3) Phil. Mag. vol. 20, pag. 417 (settembre 1910). Atti A.E.I., 1911, pag. 452. 
sinusoidale, immagina l'altra sviluppata in serie di Fourier invece che, come in quel caso, espressa dal solo primo termine di questa. Ma, per determinare i valori dei coefficienti della serie, egli ha bisogno di aver gia tracciata la curva:

$$
y=f(x) \text {, }
$$

il che implica la determinazione sperimentale di un sufficiente numero di coppie di valori corrispondenti per $x$ e per $y$, di trasformarla poi, introducendo la variabile ausiliaria $\theta$ data da:

nell'altra :

$$
x=H \operatorname{sen} \theta \text {, }
$$

$$
y=f_{1}(\theta)
$$

e di applicare a questa i noti laboriosi procedimenti grafici o meccanici di analisi armonica. Come si vede, il procedimento del Thompson obbliga a rifare per ogni materiale differente una lunga serie di operazioni fisiche e grafiche; e quando diciamo "materiale differente » abbiamo già ricordato che dobbiamo riferirci non solo a differenti campioni ma anche, per lo stesso campione, alle sue differenti condizioni presenti, non solo, ma anche passate, meccaniche e termiche.

Una applicazione ne è statà fatta da Hague o Neville ') nello studio di un campione di acciaio al silicio arrestandosi al $9^{\circ}$ armonico, per quanto anche i successivi venissero ad influire notevolmente. Precedentemente se n'era srvito l'autore della presente nota nello studio delle variazioni magnetiche prodotte colla temperatura ${ }^{2}$ ), arrivando al $5^{\circ}$ armonico.

\section{Proprietà del ciclo di isteresi.}

Scopo del presente studio è ricercare una rappresentazione analitica periodica della magnetizzazione, nei corpi ferromagnetici, in funzione del campo magnetico quando questo vari fra valori massimi uguali e di segno contrario ed il materiale sia ciclizzato. In questa prima ricerea supporremo che i limiti fra i quali varia il campo siano abbastanza estesi per poter ritenere che $i$ due rami del ciclo vengano a combaciare agli

1) « The Electrician», 13 ottubre, 1916.

2) Atti A. E.I, giugno 1911. \& Nuovo Cimento ", maggio. 1912. 
estremi. Sarà compito di ulteriore studio togliere questa restrizione per rendere la rappresentazione applicabile direttamente anche in quei casi pratici nei quali la sostanza magnetica ̀̀ cimentata sotto campi molto ristretti, nei quali appunto il combaciamento non ha luogo ma si hanno invece agli estremi dei punti angolosi.

La rappresentazione ricercata, che dovrà contenere un numero finito di termini, vogliamo sia dedotta da elementi puramente sperimentali, indipendentemente da ogni ipotesi: essa dovrà accostarsi all'andamento del fenomeno più di quello che non lo facciano il segmento di retta e la elisse ma non prosentare le difficoltà di quella di Silvanus Thompson. Dovrà essere caratterizzata per la sua forma dalle circostanze generali del fenomeno cui si riferisce e venir poi completamente determinata per mezzo di pochi elementi, facilmente accessibili, caratteristici della qualità del materiale adoperato e della ampiezza del campo al quale esso è sottoposto.

Trarremo partito dalle seguenti proprietà generali del ciclo di isteresi, rappresentato in coordinate cartesiane, proprietà che si trovano verificate per qualunque materiale ferromagnetico, purchè ciclizzato fra campi massimi eguali e di segno contrario abbastanza elevati:

a) siminetria del ciclo;

b) esistenza, per i valori massimi del campo, di due cuspidi, ciò̀ di due punti doppi nei quali le tangenti ai due rami della curva, passanti per ciascuno dei punti stessi, coincidono;

c) inclinazione delle tangenti nelle cuspidi sull'asse dei campi minore che in qualunque altro punto della curva;

d) esistenza di uno, ed un solo, punto di inflessione in ciascun ramo della curva.

In quanto alle qualità peculiari del materiale usato le riterremo caratterizzate quantitativamente, come vien fatto nella rappresentazione colla elisse, dalla mıgnetizznzione massima I raggiunta col campo m/ssimo $\mathrm{H}$ e dall'area $\mathrm{W}$ abbracciata dal ciclo, la quale misura la energia dissipata per isteresi mentre l'unità di volume del matriale percorre il ciclo stesso; anzi non considerere.no che due parametri, e cioè la magnetizzazione massima $I$ ed il rapporto $\eta=\frac{W}{4 H \bar{I}}$ fra l'area $W$ abbrac- 
eiata dal cicle e quella $4 \mathrm{HI}$ del rettangolo che ha $\mathrm{i}$ lati paralleli agli assi coordinati e che ha due vertici opposti nei vertici del ciclo (fig. 1): tale rettangolo lo chiameremo circoscritto al ciclo. Faremo uso anche del coefficente angolare $\gamma_{m}$ della tangente alla curva nelle cuspidi, ciò̀ della suscettivitò vera per

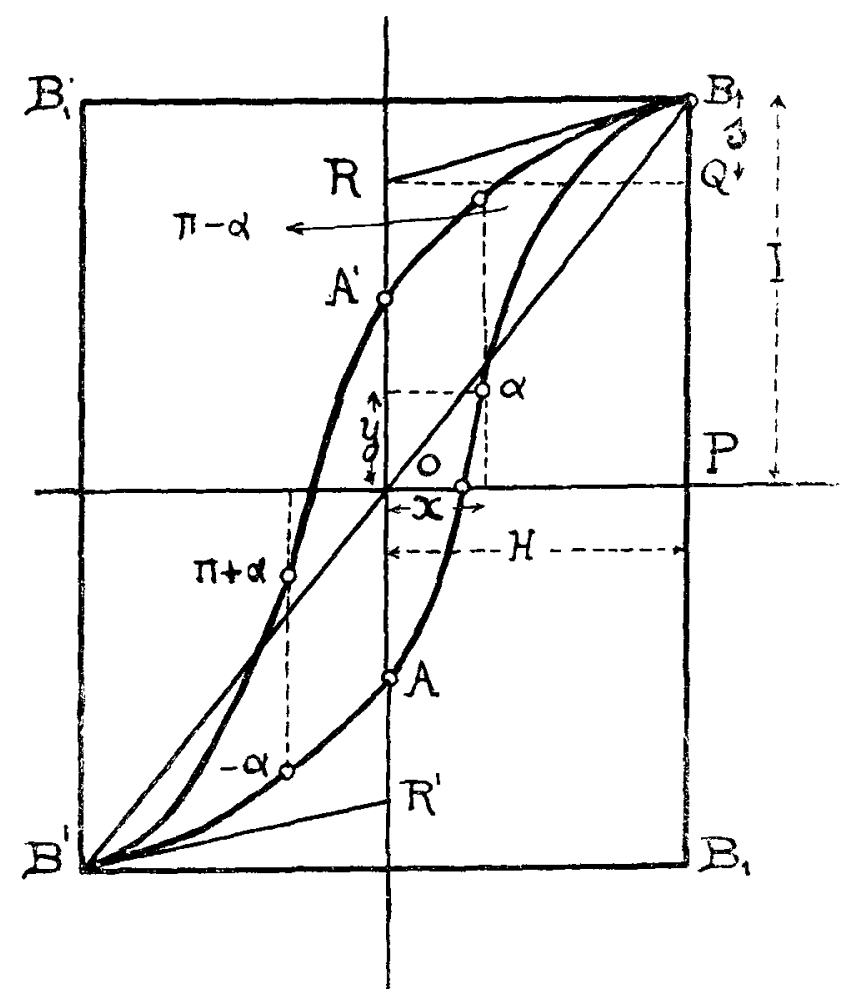

Fig. i.

campo massimo, per quanto non sia accessibile direttamente alla esperienza, ma faremo vedere come esso possa essere trascurato commettendo un errore tanto più piccolo quanto più esteso sarà il campo considerato.

Introdurremo adunque:

e) l'intensità I di magnetizzazione raggiunta col campo massimo $\mathrm{H}$; 
RAPPRESENTAZIONE ARMONICA DEL CICLO ECC. 241

f) il rapporto $\eta$ fra l'area $W$ abbraccia dal ciclo e quella $4 \mathrm{HI}$ del rettangolo circoscritto;

g) la suscettività vera $\gamma_{m}$ per campo massimo.

Si troverà che la rappresentazione analitica che andremo a proporre non sarà possibile se non con determinate limitazioni nel rapporto $\eta$ : ebbene, fisseremo che:

h) la rappresentazione sia valida per tutti i valori del rapporto $\eta$ che da zero vadano al valore più elevato possibile.

\section{Applicazione delle proprietà del ciclo.}

Indicato con $x$ il campo magnetico e con $y$ la intensità di magnetizzazione, poniamo:

$$
\begin{aligned}
& x=H \operatorname{sen} \alpha \\
& y=\Sigma Y_{m} \operatorname{sen}\left(m \alpha+\varphi_{m}\right)
\end{aligned}
$$

dove con $\mathbf{\Sigma}$ intenderemo la somma di un numero finito di termini successivi della serie di Fourier a cominciare dal primo, con $Y_{m}$ il coefficiente dell'emmesimo termine $\theta$ con $\varphi_{m}$ lo sfasamento corrispondente.

Potremo anche scrivere:

$$
y=\Sigma\left[v_{m} \operatorname{sen} m \alpha+u_{m} \cos m \alpha\right]
$$

avento fatto:

$$
\left\{\begin{array}{l}
u_{m}=\mathrm{Y}_{m} \operatorname{sen} \varphi_{m} \\
v_{m}=\mathrm{Y}_{m} \cos \varphi_{m}
\end{array}\right.
$$

Il ciclo lo immagineremo percorso nel senso A B A' $\mathrm{B}^{\prime} \mathrm{A}$ nel quale si produce effettivamente 11 fenomeno che esso rappresenta: ogni punto del ciclo sarà individuato dal corrispondente valore di $\alpha$ e, fatto corrispondere al punto $A$ il valore zero di $\alpha$, ai punti $B, A^{\prime}, B^{\prime}$ corrisponderanno rispettivamente $i$ valori $\frac{\pi}{2}, \pi$ e $\frac{3 \pi}{2}$.

Simmetria del cicln. - Dalla simmetria del ciclo emerge immediatamente che nella espressiono di $y$ devono comparire solo i termini di ordine dispari, per cui la (7), limitata ai primi di 3 armonici, potrà scriversi:

$$
y=\sum_{o}^{n}\left\{v_{2 n+1} \operatorname{sen}(2 n+1) \alpha+u_{2 n+1} \cos (2 n+1) \propto\right\} \text {. }
$$

Serie VI. $V_{0}$. XIV 
I seni e coseni degli archi multipli dispari possono mettersi, con facili trasformazioni delle formule trigonometriche ordinariamente usate, sotto la forma:

(10)

$$
\left\{\mathrm{D}_{n}=4 \sum_{h=0}^{h=n-1}(-1)^{h} 2^{2 h} \frac{(n-h)(n-h+1) \ldots(n+h)(n+h+1)}{1.2 .3 \ldots(2 h+3)} \cos ^{2 h} \alpha\right.
$$

$$
\begin{aligned}
& \operatorname{sen}(2 n+1) \alpha=(-1)^{n}\left\{1-\mathrm{C}_{n} \cos ^{2} \alpha\right\} \operatorname{sen} \alpha \\
& \cos (2 n+1) \alpha=(-1)^{n}(2 n+1)\left\{1-\mathrm{D}_{n} \cos ^{2} \alpha\right\} \cos \dot{\alpha}
\end{aligned}
$$
con:

Le espressioni $\mathrm{C}_{n} \otimes \mathrm{D}_{n}$ sono polinomi contenenti le sole potenze pari di $\cos \alpha$ e quindi, quando si faccia la sostituzione (5), di $x$ e sono di grado $2(n-1)$ rispetto alla $x$ stessa.

La (9) allora diventerà, tenuto conto della (5):

$$
y=\mathrm{E} x \pm \mathrm{F} \sqrt{\mathrm{H}^{2}-x^{2}}
$$

cen :

$$
\begin{aligned}
& \mathrm{E}=\frac{1}{\mathrm{H}} \sum_{o}^{n}(-1)^{n} v_{2 n+1}\left(1-\mathrm{C}_{n} \cos ^{2} \alpha\right) \\
& \mathrm{F}=\frac{1}{\mathrm{H}} \sum_{o}^{n}(-1)^{n}(2 n+1) u_{2 n+1}\left(1-\mathrm{D}_{n} \cos ^{2} \alpha\right)
\end{aligned}
$$

polinomi razionali interi di grado $2 n$ in $x$ contenenti le sole potenze pari di questa.

Il doppio segno che compare nella (12) sta ad indicare i due. rami della curva e precisamente il positivo quello inferiore, nel quale $\alpha$ varia $d a-\frac{\pi}{2} a+\frac{\pi}{2}$, ed il negativo quello superiore, nel quale $\alpha$ varia $d a+\frac{\pi}{2} a+\frac{3 \pi}{2}$.

L'equazione completa della curva, per eliminare il doppio segno, potrà scriversi :

$$
f(x, y)=\left[y-x \mathrm{E}-\sqrt{\mathrm{H}^{2}-x^{2}} \mathrm{~F}\right]\left[y-x \mathrm{E}+\sqrt{\mathrm{H}^{3}-x^{2}} \mathrm{~F}\right]=0 .
$$

Esistenza delle cuspidi. - Posta cosi l'equazione della curva sotto la forma $f(x, y)=0$, l'esistenza dei punti dopp della curva stessa richiederà, com'è noto, che in essi siano nulle le derivate 
parziali prime della $f(x, y)$, prese rispetto ad $x$ e ad $y$, ma non lo siano tutte e tre le derivate parziali seconde.

Dalla (14) facilmente si trae:

$$
\begin{aligned}
& \frac{\partial f}{\partial x}=-2(y-x \mathrm{E}) \frac{d(x \mathrm{E})}{d x}-2\left(\mathrm{H}^{2}-x^{2}\right) \mathrm{F} \frac{d \mathrm{~F}}{d x}+2 x \mathrm{~F}^{\mathrm{a}} \\
& \frac{\partial f}{\partial y}=2(y-x \mathrm{E}) .
\end{aligned}
$$

E poichè voglianı che i punti doppi si abbiano per $x= \pm \mathrm{H}$ ed in tali punti ̀̀ $y=x \mathrm{E}$, sarà : $\left[\frac{\partial f}{\partial y}\right]_{x= \pm \mathrm{H}}^{=0}$. Se vorremo che sia nulla anche la derivata parziale rispetto ad $x$ dovremo porre la condizione che $F$ si annulli per $x= \pm \mathrm{H}$ ciò̀ che sia verificata la equazione:

$$
\sum_{o}^{n}(-1)^{n}(2 n+1) u_{2 n+1}=0 \text {. }
$$

Dalle derivate prime ricaveremo le derivate parziali seconde le quali, ai vertici del ciclo, daranno:

$$
\begin{aligned}
& {\left[\frac{\partial^{2} f}{\partial x^{8}}\right]_{x= \pm \mathrm{H}}=2\left[\frac{d(x \mathrm{E})}{d x}\right]_{x= \pm \mathrm{H}}^{2}} \\
& {\left[\frac{\partial^{3} f}{\partial x \partial y}\right]_{x= \pm \mathrm{H}}=-2\left[\frac{d(x \mathrm{E})}{d x}\right]_{x= \pm \mathrm{H}} \quad \frac{\partial^{2} f}{\partial y^{3}}=2}
\end{aligned}
$$

Da qui si vede che la sola condizione per l'esistenza dei punti doppi sarà che sia verificata la (15) perchè, in tal caso, saranno nulle le derivate prime mentre, in ogni caso, ciò̀ qualunque sia per essere il valore di $\frac{d(x \mathrm{E})}{d x}$ ai vertici, una almeno delle derivate seconde, la $\frac{\partial^{2} f}{\partial y^{2}}$, non sarà nulla.

I coefficienti angolari delle tangenti in un punto doppio della curva $f(x, y)=0$ sono dati dalle radici $\xi_{1}$ e $\xi_{\text {g }}$ della equazione di secondo grado:

$$
\frac{\partial^{2} f}{\partial y^{2}} \xi^{2}+2 \frac{\partial^{2} f}{\partial x \partial y} \xi+\frac{\partial^{2} f}{\partial x^{2}}=0
$$

e poichè per $x= \pm H$, come si ricava dalle espressioni ora date per le derivate parziali seconde, il discriminante di questa 
equazione è nullo, risulterà che le due tangenti in ciascun punto doppio della curva coincideranno, cioè che i 2 punti doppi che essa, quando sia verificata la (1ら̆), ammette sono delle cuspidi.

Coefficiente angolare della tangente. - Dalla detta equazione di secondo grado ora scritta risulta ehe il coefficiente angolare della tangente in ciascuna delle cuspidi sarà :

$$
\begin{aligned}
& \gamma_{m}=\left[\frac{d(x \mathrm{E})}{d x}\right]_{x= \pm \mathrm{H}} \text { ossia : } \\
& \gamma_{m}=\frac{1}{\mathrm{H}} \sum_{o}^{n}(-1)^{n}(2 n+1)^{2} v_{2}+1 .
\end{aligned}
$$

Indicato con $\gamma$ il coefficiente angolare $\frac{d y}{d x}$ della tangente alla curva in un punto generico di essa, la condizione espressa alla lettera (c), che le tangenti nelle cuspidi siano meno inclinate sull'asse dei campi che in qualsiasi altro punto della curva, si traduce nell'altra che $\gamma-\gamma_{m}$ debba conservarsi sempre positivo in tutto l'intervallo, salvo agli estremi dove si annulla.

Partendo dalla (9) e tenendo conto della (5) abbiamo:

$$
\begin{aligned}
& \gamma=\frac{d y}{d x}=\frac{d y}{d \alpha} \frac{d \alpha}{d x}=\frac{1}{H \cos \alpha}\left\{\sum_{o}^{n}(2 n+1) v_{2 n+1} \cos (2 n+1) \alpha\right. \\
& \left.-\sum_{0}^{n}(2 n+1) u_{2 n+1} \operatorname{sen}(2 n+1) \alpha\right\} .
\end{aligned}
$$

Ricordando le (10) questa diviene:

$$
\begin{aligned}
& \gamma=\frac{1}{H \cos \alpha}\left\{\sum_{0}^{n}(-1)^{n}(2 n+1)^{2} v_{2 n+1-1}\left(1-\mathrm{D}_{n} \cos ^{2} \alpha\right) \cos \alpha\right. \\
& \left.-\sum_{0}^{n}(-1)^{n n}(2 n+1) u_{2 n+-1}\left(1-\mathrm{C}_{n} \cos ^{2} \alpha\right) \operatorname{sen} \alpha\right\}
\end{aligned}
$$

da cui, per le (16) e (15):

$$
\begin{aligned}
& \mathrm{H}\left(\gamma-\gamma_{m}\right)=\cos \alpha\left[-\sum_{o}^{n}(-1)^{n}(2 n+1)^{2} v_{2, n+1} \mathrm{D}_{n} \cos \alpha\right. \\
& \left.+\sum_{n}^{n}(-1)^{n}(2 n+1) u_{2 n+1} \mathrm{C}_{n} \operatorname{sen} \alpha\right] .
\end{aligned}
$$


RAPPRESENTAZIONE ARMONICA DEL CICLO ECC. 245

La espressione, per il fatto che porta in evidenza il fattore $\cos \alpha$, si annulla per $x= \pm H$, come, del resto, deriva dalla stessa definizione di $\gamma_{m}$. Perchè poi non cambi mai segno nell'intervallo occorrerà che, nell' intervallo stesso, non cambi mai segno la espressione fra parentesi quadra. Ma in questa, agli estremi, il $10^{\circ}$ termine si annulla, mentre il $2 .^{\circ}$ acquista valori eguali e di segno contrario poichè $\mathrm{C}_{n}$, definito dalla 1 . $^{\mathrm{a}}$ delle (11), non cambia segno essendo funzione razionale di $\cos ^{2} \alpha$ : occorrerà quindi che questo $2 .^{\circ}$ termine sia, agli estremi, identicamente nullo, ciò̀ che si abbia:

$$
\sum_{o}^{n}(-1)^{n}(2 n+1) u_{2 n+1} \mathrm{C}_{n o}=0
$$

avendo indicato con $\mathrm{C}_{n o}$ il termine indipendente $\mathrm{da} \cos ^{2} \alpha$ nella espressione di $\mathrm{C}_{n}$.

E facile vedere dalla $1 .{ }^{2}$ delle (11) che, arrestandoci con $n$ ai valori $0,1,2,3 \ldots$, la espressione $C_{n o}$ assumerà rispettivamente i valori:

$$
\mathrm{C}_{00}=0, \quad \mathrm{C}_{10}=4, \quad \mathrm{C}_{20}=12, \quad \mathrm{C}_{30}=24 \ldots
$$

per cui, nei vari casi, il primo membro della (18), che per $n=o$ è identicamente nullo, diviene rispettivamente:

$$
\left\{\begin{aligned}
& \text { per } n=1 \quad u_{3}=0 \\
& x_{3} u_{3}-5 u_{5}=0 \\
&=3 u_{3}-5 u_{5}+14 u_{7}=0 \\
& \ldots \ldots \ldots \ldots
\end{aligned}\right.
$$

Dalla condizione (c), della quale ci siamo ora occupati, deriva immediatamente che la tangente in ciascuna cuspide ai due rami della curva si lascia questi dalla stessa parte e che quindi le 2 cuspidi sono di seconda specie, come appunto quelle che si hanno nei cicli sperimentali di isteresi.

Indicato con $\gamma_{r}$ il valore che assume il coefficiente angolare della tangente alla curva nei punti corrispondenti al magnetismo residuo, ciò in $\mathrm{A}$ ed $\mathrm{A}^{\prime}$ della fig. 1 pei auali $\dot{e} \alpha$ rispettivamente uguale a o ed a $\pi$, la (17) darà:

$$
k=\mathrm{H}\left(\gamma_{r}-\gamma_{m}\right)=-\sum_{0}^{n}(-1)^{n}(2 n+1)^{2} v_{2 n+1} \mathrm{D}_{n 1}
$$


dove s' $\dot{e}$ indicato con $D_{n 1}$ ciò che diventa $D_{n}$ quando si ponga in esso $\cos ^{2} \alpha=1$.

Analogamente a quanto $s^{\prime} \dot{\theta}$ fatto precedentemente avremo che, arrestandoci con $n$ ai valori $0,1,2,3 \ldots$, la $D_{n 1}$ assumerà i valori:

$$
0, \frac{4}{3}, \frac{4}{5}, \frac{8}{7}, \ldots
$$

Alle varie proprietà del ciclo si può soddisfare, come vedremo, assegnando i valori di 6 parametri, cioè prendendo per $n 3$ valori: se per questi si prenderanno 0,1 , e 2 la (17) diventerà :

$$
\begin{aligned}
& \mathrm{H}\left(\gamma-\gamma_{m}\right)=\cos \alpha\left[\left\{-v_{1} \mathrm{D}_{\mathrm{o}}+9 v_{\mathrm{s}} \mathrm{D}_{1}-25 v_{\mathrm{s}} \mathrm{D}_{2}\right\} \cos \alpha+\right. \\
& \left.+\left\{u_{1} \mathrm{C}_{0}-3 u_{\mathrm{s}} \mathrm{C}_{1}+5 u_{\mathrm{g}} \mathrm{C}_{2}\right\} \operatorname{sen} \alpha\right] \\
& =4 \cos \alpha\left[\left\{3 v_{3}-25 v_{5}\left(1-\frac{4}{5} \cos ^{2} \alpha\right)\right\} \cos \alpha\right. \\
& \left.+\left\{-3 u_{3}+5 u_{5}\left(3-4 \cos ^{2} \alpha\right)\right\} \operatorname{sen} \alpha\right] \\
& =4 \cos \alpha\left[\left\{3 v_{3}-5 v_{5}-20 v_{5} \operatorname{sen}^{3} \alpha\right\} \cos \alpha\right. \\
& \left.+\left\{-3\left(u_{3}-5 u_{5}\right)-20 u_{5} \cos ^{2} \alpha\right\} \operatorname{sen} \alpha\right]
\end{aligned}
$$

e, ricordando che arrestandoci con $n$ al valore 2, $\dot{e}$ :

$$
u_{3}-5 u_{\mathrm{s}}=0 \text {, }
$$

potremo scrivere:

$\mathrm{H}\left(\gamma-\gamma_{m}\right)=4\left[\left(3 v_{3}-5 v_{b}\right)-20 v_{3} \operatorname{sen}^{2} \alpha-20 u_{\mathrm{g}} \operatorname{sen} \alpha \cos \alpha\right] \cos ^{2} \alpha$, e:

$$
k=\mathrm{H}\left(\gamma_{r}-\gamma_{m}\right)=4\left(3 v_{3}-\tilde{\mathrm{s}} v_{5}\right),
$$

per cui :

$\mathrm{H}\left(\gamma-\gamma_{m}\right)=\left[k-80 v_{6} \operatorname{sen}^{2} \alpha-80 u_{5} \operatorname{sen} \alpha \cos \alpha\right] \cos ^{2} \alpha$.

$$
\text { Ponendo: }
$$

$$
3 v_{3}-5 v_{y}=10 m
$$

sarà :

$\mathrm{H}\left(\gamma-\gamma_{m}\right)=40\left[m-2 v_{\mathrm{s}} \operatorname{sen}^{2} \alpha-2 u_{\mathrm{g}}\right.$ sen $\left.\alpha \cos \alpha\right] \cos ^{2} \alpha$.

Sostituendo infine alla variabile $\alpha$ la $x$ data dalla (5) avremo:

$$
\begin{aligned}
& \frac{1}{40} \mathrm{H}^{\mathrm{s}}\left(\gamma-\gamma_{m}\right)=m \mathrm{H}^{2}\left(\mathrm{H}^{2}-x^{2}\right)- \\
& -2 v_{\mathrm{v}} x^{2}\left(\mathrm{H}^{2}-x^{2}\right) \pm 2 u_{5} x\left(\mathrm{H}^{2}-x^{2}\right)^{3} \mathrm{~s}
\end{aligned}
$$


Punto di inflessione e condizioni per la sua esistenza ed unicità. - La curva sperimentale ammette un punto di inflessione, ed uno solo, in ciascun ramo. Ciò si traduce, salvo l'esame delle derivate superiori, nella condizione che si abbia in uno ed uno solo punto di ciascun ramo della curva, cioè, in ciascun ramo, per $x$ compreso fra $\pm \mathrm{H}$ :

$$
\frac{d^{2} y}{d x^{2}}=0, \quad \text { ossia } \frac{d \gamma}{d x}=0 .
$$

Derivando la (21) rispetto ad $x$ avremo:

$$
\frac{1}{80} \mathrm{H}^{5} \frac{d \gamma}{d x}=-x\left\{m \mathrm{H}^{2}+2 v_{5}\left(\mathrm{H}^{2}-2 x^{2}\right)\right\} \pm u_{5} / \mathrm{H}^{2}-x^{8}\left\{\mathrm{H}^{2}-4 x^{2}\right\}
$$

e la condizione $\frac{d \gamma}{d x}=o$ sarà verificata per quei valori di $x$ che annulleranno la espressione:

$$
\Theta=x^{3}\left\{m \mathrm{H}^{2}+2 v_{3}\left(\mathrm{H}^{3}-2 x^{2}\right)\right\}^{2}-u_{\mathrm{⿺}}^{2}\left(\mathrm{H}^{2}-x^{2}\right)\left(\mathrm{H}^{3}-4 x^{3}\right)^{2} \text {. }
$$

Ponendo:

$$
x^{2}=\mathrm{H}^{2} z,
$$

eseguendo i calcoli ed ordinando, la $\Theta=-0$ assumerà la forma:

$$
\begin{aligned}
& 16\left(u_{\mathrm{s}}^{2}+v_{\mathrm{s}}^{2}\right) z^{3}-8\left(3 u_{\mathrm{s}}^{3}+2 v_{\mathrm{s}}^{2}+m v_{\mathrm{s}}\right) z^{3}+ \\
& +\left(9 u_{5}^{2}+4 v^{2}+4 m v_{5}+m^{2}\right) z-u_{5}^{2}=o
\end{aligned}
$$

cioè quella di una equazione completa di $3 .^{\circ}$ grado.

Al variare di $x$ fra $\pm H$ corrisponde la variazione di $z$ fra 0 ed 1: se esiste dunque su ciaseun ramo della curva un punto di inflessione ed uno solo, dovrà annullarsi $\theta$, al passaggio di $z$ fra 0 ed 1 , una ed una sol volta. Per decidere se ciò succede occorre, come $\dot{e}$ noto, messa la equazione $\Theta=0$ sotto la forma:

$$
w^{3}+p w+q=o,
$$

esaminare la espressione:

$$
\mathrm{D}=4 p^{3}+27 q^{2}
$$

che compare nella formola risolutiva. 
Tale espressione, fatte le opportune sostituzioni e riduzioni, diviene:

$$
\mathrm{D}=-\frac{\left(m+v_{\mathrm{b}}\right)^{2}}{32^{2}\left(u_{\mathrm{g}}^{2}+v_{\mathrm{g}}^{2}\right)^{4}} u_{\mathrm{g}}^{\mathrm{g}}
$$

$$
\left\{27 u_{5}^{4}-18\left(m^{2}-2 m v_{5}-2 v_{5}^{2}\right) u_{5}^{2}-\left(m+2 v_{5}\right)^{3}\left(m-2 v_{5}\right)\right\}
$$

e sotto tale forma si presta bene alla discussione.

Vedremo in seguito (33) come $u_{3}$ sia proporzionale all'area abbracciata dal ciclo per cui quando tale area sarà nulla lo sarà pure la $u_{\mathfrak{s}}$ e quindi la D; d'altro lato, noi abbiamo stabilito (h) che la rappresentazione analitica che andiamo a cercare debba essere valida anche quando l'area abbracciata si annulla.

Consideriamo quattro casi distinti:

$1 .^{\circ}$ caso $: m+v_{5}=o$, e quindi $\mathrm{D}=0$. Le 3 radici della equazione di $3 .^{\circ}$ grado $\Theta=0$ saranno, come ̀̀ noto, tüte $\mathrm{e}$ tre reali e due di esse uguali fra loro.

Se vorremo adunque che $\Theta$ si annulli, nell' in tervallo da zero ad uno, una ed una sola volta, occorrerà che la radice semplice sia compresa nell' intervallo e non lo sia invece quella doppia. La prima condizione per $u_{a}=0$ è soddisfatta poichè in questo caso la (23) ammette la radice $z=0$. Tolta questa radice la (23) diventa:

$$
16 v_{5}^{2} z^{2}-8 v_{5}\left(2 v_{5}+m\right) z+\left(2 v_{5}+m\right)^{2}=0
$$

la quale ammette la radice doppia:

$$
z_{1}=z_{2}=\frac{m+2 v_{5}}{4 v_{s}}=\frac{1}{4}
$$

compresa anch'essa nell'intervallo. Il caso adunque nel quale si abbia $m+v_{\mathrm{s}}=0$, ossia $3 v_{3}+5 v_{\mathrm{v}}=0$, va escluso.

$2 .^{\circ}$ caso: $m+v_{5} \neq 0, u_{6}=0$ e quindi ancora $\mathrm{D}=0$.

E facile vedere che qualora si scelga:

$$
2 \bar{l}_{5} \leq \bar{m}
$$

avendo indicato con $\overline{\vec{i}}_{5}$ ed $\bar{m}$ i valori assoluti di $v_{\mathrm{s}}$ ed $m$, la radice doppia sarà esterna all'intervallo od, al più, in uno degli estremi di questo e precisamente minore di zero od al più uguale quando $v_{\mathrm{g}}$ avrà segno opposto a quello di $m$ e maggiore di 1 od al più uguale quando avrà lo stesso segno. 
RAPPRESENTAZIONE ARMONICA DEL CICLO ECC. 249

3. caso: $m+v_{5} \neq o, u_{5} \neq o$ e $\mathrm{D}=o$. L'essere $\mathrm{D}=o$ senza esserlo $m+v_{5}$ ed $u_{5}$ porta che sia nulla la espressione entro parentesi nella (24) cioè che $u_{\mathfrak{s}}$ sia tale da soddisfaro alla equazione:

$$
\begin{aligned}
& 27 u^{4}-18\left(m^{2}-2 m v_{5}-2 v_{5}^{2}\right) u^{2}{ }_{5}- \\
& -\left(m+2 v_{5}\right)^{3}\left(m-2 v_{5}\right)=o .
\end{aligned}
$$

$\mathrm{Si}$ osserva che il termine noto di questa equazione di secondo grado in $u^{2}{ }_{\mathrm{b}}$ è certamente negativo o nullo poichè, dovendo essere possibile la rappresentazione anche nel $1 .^{\circ}$ caso, sarà verificata la disugua ed $u_{\text {sa }}^{2}$ avranno segno contrario, a meno che una di esse non sia nulla. D'altra parte, poichè $u_{5}$, come s' è accennato prima e vedremo meglio in seguito, è proporzionale all'area del ciclo e quindi è reale, $u_{5}^{2}$ deve essere positivo e però delle due radici :

$$
\begin{aligned}
& u_{\mathrm{s}}^{2}=\frac{1}{27}\left\{9\left(m^{2}-2 m v_{\mathrm{b}}-2 v_{\mathrm{s}}^{2}\right) \pm\right. \\
& \left. \pm \sqrt{81\left(m^{2}-2 m v_{5}-2 v_{5}^{2}\right)^{2}+27 \overline{\left(m+2 v_{5}\right)^{3}\left(m-2 v_{5}\right)}}\right\},
\end{aligned}
$$

ossia :

$$
u_{\mathrm{s}}^{2}=\frac{1}{9}\left\{3\left(m^{2}-2 m v_{\mathrm{b}}-2 v_{\mathrm{b}}^{3}\right) \pm 2\left(m-v_{\mathrm{s}}\right) \sqrt{ }\left(\overline{\left(m^{2}-v_{\mathrm{b}}^{2}\right)}\right\},\right.
$$

solo la positiva $u_{{ }_{51}}^{2}$ dovrà considerarsi.

4. ${ }^{\circ}$ caso: $m+v_{\mathrm{s}} \neq 0, u_{\mathrm{s}} \neq o, \mathrm{D}>0$.

Mentre $u_{5}^{2}$ varia da $o$ ad $u_{51}^{2}$ la $\mathrm{D}$ si mantiene sempre positiva poichè ha segno opposto del trinomio primo membro della (26) che è negativo per $u_{5}^{2}$ compreso fra $u_{52}^{2}$ ed $u_{51}^{2} \mathrm{e}$ quindi fra $o$ ed $u_{51}^{2}$. Ma, come è noto dalla teoria delle equazioni di $3 .^{\circ}$ grado, per $\mathrm{D}>0$ una sola delle radici della equazione è reale, quindi fino a quando $u_{\mathrm{g}}^{2}$ rimarrà entro il detto intervallo la rappresentazione cercata ammetterà un solo punto di inflessione in ciascun ramo, salvo sempre il caso che l'esame delle derivate superiori non faccia escludere anche questo.

Abbiamo posto $(g)$ la condizione che la rappresentazione valga quando il ciclo racchiuda un'area che dall'essere nulla possa crescere quanto più sia possibile: dovremo adunque fare 
in modo che $u_{51}^{2}$ assuma il più elevato valore. E poichè, come $\dot{\theta}$ facile vedere, $u^{2}{ }_{51} \dot{\theta}$ wecrescente con $v_{5}$, la condizione sarà raggiunta quando si prenda per questo parametro il suo più piccolo valore, algebricamente parlando, che soddisfa alla (25), ossia quando si faccia:

$$
2 v_{\mathrm{s}}=-m
$$

Sostituendo ad $m$ il suo valore dato dalla (20) avremo:

$$
v_{3}+\tilde{5} v_{\mathrm{s}}=0 \text {. }
$$

Con questo valore $2 v_{5}=-m$ le radici $u_{{ }_{52}}^{2}$ ed $u_{{ }_{11}}^{2}$ diven. tano rispettivamente:

$$
u^{2}{ }^{2}=o \text { ed } u^{2}{ }^{2}=m^{2}=4 v_{5}^{2},
$$

il che sta ad indicare che, fino a quando $u^{2}{ }_{3}$ soddisfa alla condizione :

$$
0 \leq u^{2} \leq 4 v^{y}{ }_{5},
$$

la (25) ammetterà una sola radice reale $z_{0}$ fra 0 ed 1 . In altre parole, entro tali limiti, in due soli punti $x_{1}=\mathrm{H} \sqrt{z_{0}}, x_{2}=-\mathrm{H} \sqrt{z_{0}}$ dell'intervallo $\pm \mathrm{H}$ si annullerà $\frac{d \gamma}{d x}=\frac{d^{2} y}{d x^{2}}$.

Introdotto il valore di $v_{5}$, dato dalla (27), nella (22) otteniamo:

$$
\frac{d \underline{\gamma}}{d x}=\frac{d^{2} y}{d x^{2}}=-{ }_{\mathrm{H}^{\mathrm{B}}}^{80}\left\{2 m x^{3} \pm u_{\mathrm{s}} \sqrt{\mathrm{H}^{2}-x^{2}}\left(\mathrm{H}^{2}-4 x^{2}\right)\right\}
$$

dalla quale, calcolando le derivate superiori, si vede che per $u_{\mathrm{b}}$. differente da zero i valori di $x$ che annullano la derivata seconda della $y$ non annullano la terza, mentre per $u_{5}=0$ ai annullano anche la terza e quarta ma non la quinta, ossia che in ogni caso la più bassa derivata della $y$ rispetto alla $x$, superiore alla prima, che non si annulla nei punti dove si annulla la derivata seconda, ̀ di ordine dispari : e però tali punti sono di inflessione. 
Intensità massima di magnetizzazione. - Se nella (9) facciamo $\alpha= \pm \frac{\pi}{2}$ ed indichiamo con $\pm I$ il valore massimo che assume l'ordinata corrispondente, abbiamo:

$$
I=\sum_{0}^{n}(-1)^{n} v_{2 n+1}
$$

la quale ci esprimerà la intensità massima raggiunta dalla magnetizzazione.

Area del ciclo. - In quanto all'area W essa sarà data da:

$$
\mathrm{W}=\int y d x
$$

colla integrazione estesa a tutto il ciclo chiuso di magnetizzazione ed immaginando il ciclo stesso percorso in senso destrorso. Poichè però, di fatto, il ciclo, nel fenomeno della magnetizzazione, è percorso nel senso opposto, per avere l'area, che $\grave{e}$ essenzialmente positiva, dovremo prendere l' integrale col segno contrario.

Sostituendo alla variabile $x$ la $\alpha$ data dalla (5) ed alla $3 J$ la sua espressione (9) potremo scrivere:

$$
\begin{aligned}
& \mathrm{W}=-\mathrm{H} \int_{0}^{2 \pi} y \cos \alpha d \alpha= \\
& =-\mathrm{H}\left[\sum_{0}^{n} v_{2 n+1} \int_{0}^{2 \pi} \operatorname{sen}(2 n+1) \alpha \cos \alpha d \alpha+\right. \\
& \left.+\sum_{o}^{n} u_{2 n+1} \int_{0}^{2 \pi} \cos (2 n+1) \alpha \cos \alpha d \alpha\right]
\end{aligned}
$$

e poiché, come è noto, tutti gli integrali della prima somma sono nulli e lo sono pure quelli della seconda, tranne che quello corrispondente ad $n=0$, il quale ha per valore $\pi$, avremo:

$$
\mathrm{W}=-\pi \mathrm{H} u_{\mathbf{\imath}} \text {. }
$$

Quest' ultima potrà scriversi :

$$
u_{1}=-\frac{4}{\pi} \eta I
$$


nella quale ricordiamo che si $\dot{e}$ indicato con $\eta$ il rapporto $\frac{W}{4 H T}$ fra l'area abbracciata dal ciclo e quella del rettangolo circoscritto.

Suscettività vera agli estremi. - La suscettività vera in un punto qualsiasi del ciclo è data dal coefficiente angolare della tangente alla curva nel punto stesso. Quella agli estremi sarà il coefficiente angolare $\gamma_{m}$ nelle cuspidi già espresso nella (11).

\section{Determinanzione delle $u$ e delle $v$.}

Colle considerazioni suesposte siamo cosi venuti a stabilire sei equazioni delle quali tre fra le $u$ :

(31) $u_{1}$

$$
\begin{aligned}
& u_{1}-3 u_{3}+5 u_{5}=0 \text { (per l'esistenza delle cuspidi) } \\
& u_{3}-5 u_{5}=0 \text { (perchè la tangente sia meno in- } \\
& \text { clinata agli estremi che in tutti } \\
& \text { gli altri punti) } \\
& u_{1}=-\frac{4}{\pi} \eta \mathrm{I} \text { (assegnazione del rapporto } \\
& \text { fra l'area del ciclo o quella del } \\
& \text { rettangolo circoscritto) }
\end{aligned}
$$

e 3 fra le $v$ :

$$
\begin{aligned}
& \text { (16) } v_{1}-9 v_{3}+25 v_{5}=\mathrm{H} \gamma_{m} \begin{array}{l}
\text { (assegnazione del coefficiente } \\
\text { angolare della tangente agli } \\
\text { estremi) }
\end{array} \\
& v_{3}+5 v_{5}=0 \begin{array}{r}
\text { (per l'esistenza del punto di in- } \\
\text { flessione in ciascun ramo per } \\
\text { area variabile da zero al va- } \\
\text { lore più elevato possibile). }
\end{array} \\
& \text { (30) } v_{1}-v_{3}+v_{5}=\mathrm{I} \quad \begin{array}{c}
\text { (assegnazione della magnetizza- } \\
\text { zione massima). }
\end{array}
\end{aligned}
$$

Oltre alle dette equazioni sarà per la esistenza del punto unico di inflessione, in ciascun ramo, da verificare la disuguaglianza (29) ciò, in valore assoluto:

$$
u_{5} \leq 2 v_{\mathrm{s}} \text {. }
$$


Dalle 6 equazioni, ponendo $s=\mathrm{H} \gamma_{m}$, si ricava:

(33)

$$
\begin{aligned}
& u_{1}=-\frac{4}{\pi} \eta \mathrm{I} \\
& u_{3}=-\frac{2}{\pi} \eta \mathrm{I} \\
& u_{3}=-\frac{2}{5 \pi} \eta \mathrm{I} \\
& v_{1}=\frac{1}{32}(35 \mathrm{I}-3 s)=\frac{35}{32}\left(1-0,0857 \frac{s}{\mathrm{I}}\right) \mathrm{I} \\
& v_{3}=\frac{5}{64}(\mathrm{I}-s)=\frac{5}{64}\left(1-\frac{s}{\mathrm{I}}\right) \mathrm{I} \\
& v_{5}=-\frac{1}{64}(\mathrm{I}-s)=-\frac{1}{64}\left(1-\frac{s}{\mathrm{I}}\right) \mathrm{I}
\end{aligned}
$$

che danno le sei costanti della espressione (9) per mezzo dei due parametri definitivi $\eta$ ed I e del parametro provvisorio $s=\mathrm{H} \gamma_{m}$, dove $\gamma_{m} \dot{\theta}$ il coefficiente angolare della tangente nelle cuspidi: vedremo in seguito come potrà assegnarsi il valore di quest'ultimo.

Dalla disuguaglianza (32) poi, introducendo i valori assoluti di $u_{5}$ e $v_{5}$, avremo:

ossia :

$$
\frac{64 \eta}{5 \pi} \leq 1-\frac{s}{\mathrm{I}}
$$

$$
\eta \leq 0,245\left(1-\frac{s}{\mathrm{I}}\right)
$$

la quale, ricordando il significato di $\eta$, ci avverte, come s'era accennato precedentemente $(h)$, che, per poter usare la rappresentazione data dalle (5) e (9), dovrà l'area abbracciata dal ciclo non superare una certa frazione di quella del rettangolo circo. scritto.

Il rapporto $\frac{s}{\mathrm{I}}$ ha un significato fisico notevole, come apparisce dalla fig. 1. Esso è infatti :

$$
\frac{s}{\mathrm{I}}=\frac{\mathrm{H} \gamma_{m}}{\mathrm{I}}=\frac{\gamma_{m}}{\mathrm{I}: \mathrm{H}}=\frac{\gamma_{m}}{\gamma_{o}}=\frac{t g \mathrm{BRQ}}{t g \mathrm{BOP}}
$$


ossia è uguale al rapporto fra la suscettività vera $\gamma_{m}$ al vertice del ciclo e quella media $\gamma_{0}$ lungo tutto il ciclo (1). Col crescer $\theta$ del campo il detto rapporto tende a sparire e veramente si annulla quando sia raggiunta la saturazione magnetica. In molti casi pratici potrà, senz'altro, essere trascurato ed è da osservarsi come l'errore che tale trascuranza verrà ad introdurre, mentre non avrà alcuna influenza per le $u$, sarà minore per la $v_{1}$ che per le altre $v$ poichè in quella il detto rapporto è moltiplicato per un cuefficiente molto inferiore all' unità, mentre in queste il coefficiente è l'unità stessa. Quando il rapporto $\frac{s}{I}$ non possa trascurarsi se ne determinerà il valore mediante esperienze di magnetizzazione statica ottenuta nelle stesse circostanze nelle quali si sarà ottenuta la magnetizzazione dinamica che ci saremo proposti di rappresentare.

Il magnetismo residuo indicato dalla rappresentazione si otterrà facendo nella (7) $\alpha$ uguale a zero od a $\pi$. Tutti i termini contenenti $i$ seni si annulleranno mentre $i$ coseni assumeranno il valore \pm 1 . Avremo cosi:

$$
\mathrm{I}_{r}= \pm\left(u_{1}+u_{3}+u_{\mathrm{s}}\right)
$$

ed, in valore assoluto, sostituendo $\mathrm{i}$ valori trovati per le $u$ :

$$
\mathrm{I}_{r}=\frac{32}{5 \pi} \eta \mathrm{I} \text {. }
$$

Per $\eta=0$ sarà, come già si sapeva, $\mathrm{I}_{r}=0$, e per $\eta$ massimo, ciò̀ per: $\eta=\frac{5}{6} \frac{\pi}{4}\left(1-\frac{\mathrm{s}}{\mathrm{I}}\right)$ avremo:

$$
\mathrm{I}_{r}=\frac{1}{2}(\mathrm{I}-\mathrm{s}) \text {. }
$$

Non va confuso queste magnetismo residuo indicato con quello effettivo dal quale potrà differire.

\section{Rappresentazione armonica.}

Da quanto abbiamo esposto risulta che, supponendo trascurabile la $s$ di fronte alla $I$, cioè supponendo che sensibilmente le tangenti alle cuspidi siano parallele all'asse dei campi, la

I) V. questo lavoro. Nota (1) a pag. 237. 
RAPPRESENTAZIONE ARMONICA DEL CICLO ECC. 25̃ espressione della magnetizzazione, quando si intenderà il campo dato dalla $x=\mathrm{H}$ sen $\alpha$, assumerà la forma:

ossia :

$$
\begin{aligned}
& y=\frac{1}{64}[\{70 \operatorname{sen} \alpha+5 \operatorname{sen} 3 \alpha-\operatorname{sen} 5 \alpha\}- \\
& \left.-\frac{2 \cdot 64}{5 \pi} \eta\{10 \cos \alpha+5 \cos 3 \alpha+\cos 5 \alpha\}\right] \mathrm{I}
\end{aligned}
$$

$$
y=\frac{1}{64}\left(\mathrm{X}-c \mathrm{X}^{\prime}\right) \mathrm{I}
$$

con

$$
c=\frac{2 \cdot 64}{5 \pi} \eta=8,15 \eta_{1}
$$

variabile da 0 a 2 ,

e con:

$$
\begin{aligned}
& X=70 \operatorname{sen} \alpha+5 \operatorname{sen} 3 \alpha-\operatorname{sen} 5 \alpha \\
& X^{\prime}=10 \cos \alpha+5 \cos 3 \alpha+\cos 5 \alpha .
\end{aligned}
$$

La (40) potrà facilmente essere messa sotto l'altra forma:

$$
\begin{aligned}
& y=\frac{1}{64}\left[a_{1} \operatorname{sen}\left(\alpha+\varphi_{1}\right)+a_{3} \operatorname{sen}\left(3 x+\varphi_{3}\right)+\right. \\
& \left.+a_{5} \operatorname{sen}\left(5 \alpha+\varphi_{5}\right)\right] \mathrm{I}
\end{aligned}
$$

dove tanto i coefficienti $a_{1}, a_{3}, a_{5}$ che determinano le ampiezze rispettive dei singoli armonici, quanto gli sfasamenti $\varphi_{1}, \varphi_{3}, \varphi_{5}$ rispetto al campo, non dipenderanno se non dal rapporto $r_{i}$. Essi saranno:

$$
\begin{array}{ll}
a_{1}=10 \sqrt{49 \dot{r} 66 \eta^{2}} & t g \varphi_{1}=-1,16 \eta \\
a_{3}=5 \sqrt{1+66 \eta^{2}} & \operatorname{tg} \varphi_{3}=-8,15 \eta \\
a_{\mathrm{s}}=\sqrt{1+66 \eta^{2}} & \operatorname{tg} \varphi_{\mathrm{s}}=8,15 \eta .
\end{array}
$$

Sviluppando nella (40) i seni e coseni degli archi multipli in potenze dei seni e coseni dell'areo semplice, potremo anche scrivere:

$$
y=\frac{1}{4}\left[\operatorname{sen} \alpha\left(5-\operatorname{sen}^{4} \alpha\right)-\frac{128 \eta}{5 \pi} \cos ^{5} \alpha\right] \mathbf{I}
$$

e sostituendo alla variabile $\propto$ la $x$ :

$$
y=\frac{1}{4 \mathrm{H}^{5}}\left[x\left(5 \mathrm{H}^{4}-x^{4}\right) \pm \frac{128 \eta}{5 \pi}\left(\mathrm{H}^{4}-x^{3}\right)^{\frac{5}{2}}\right] \mathrm{I}
$$


dove del doppio segno il - si riferisce al ramo inferiore della curva ed il + al superiore. Di queste tre forme prenderemo, caso per caso, quella che più farà comodo.

Ritornando alla (41) si riportano nella tavola seguente i valori che assumono, al variare di $\alpha$ di 10 in 10 gradi, le $X$ ed $\mathrm{X}^{\prime}$ od $\mathrm{i}$ corrispondenti valori di $\mathrm{X}-c \mathrm{X}$ per $c$ uguale a $0 ; 1 ; 2 ; 3$, ossia per $\eta$ uguale a $0 ; 0,123 ; 0,24 \check{5} ; 0,368$.

\begin{tabular}{|c|c|c|c|c|c|c|c|c|c|c|}
\hline \multirow{2}{*}{$\underline{\alpha}$} & \multicolumn{2}{|c|}{$\mathbf{X}^{\prime}$} & \multicolumn{2}{|c|}{$\begin{array}{c}\mathrm{x} \\
\eta=0\end{array}$} & \multicolumn{2}{|c|}{$\begin{array}{c}X-X^{\prime} \\
\eta=0,123\end{array}$} & \multicolumn{2}{|c|}{$\begin{array}{l}x-2 x \\
\eta=0,245\end{array}$} & \multicolumn{2}{|c|}{$\begin{array}{c}x-3 X^{\prime} \\
\eta=0,368\end{array}$} \\
\hline & + & 16.- & & 0.- & - & 16. - & - & 32.- & - & 48.- \\
\hline $10^{\circ}$ & + & 14.82 & + & 1389 & - & 0.93 & - & 15.76 & - & 30.58 \\
\hline $20^{\circ}$ & + & 11.62 & + & 27.28 & + & 15.66 & + & 4.04 & - & 7.58 \\
\hline $30^{\circ}$ & + & 7.79 & + & 39.500 & + & 31.71 & + & 23.91 & + & 16.12 \\
\hline $40^{\circ}$ & + & 4.22 & + & 48.98 & + & 44,76 & + & 40.54 & + & 36.32 \\
\hline $50^{\circ}$ & + & 1.76 & + & 5706 & + & 55.30 & + & 53.55 & + & 51.79 \\
\hline 60 & + & 0.50 & + & 61.49 & + & 60.99 & + & 6049 & + & 59.99 \\
\hline 70 & + & 0.07 & + & 63.45 & 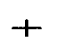 & 63.38 & + & 6331 & + & 63.20 \\
\hline 80 & + & 0.00 & + & 6396 & + & 63.96 & + & 63.96 & + & 63.96 \\
\hline 90 & + & 0. - & + & 64.- & + & 64.- & + & 64.- & + & 64.- \\
\hline $100^{\circ}$ & - & 0.00 & + & 63.96 & + & 63.96 & + & 6396 & + & 63.97 \\
\hline $110^{\circ}$ & - & 0.07 & + & 63.45 & + & 63.52 & + & 63.60 & + & 63.67 \\
\hline $120^{\circ}$ & - & 0.50 & + & 61.49 & + & 61.99 & + & 62.49 & + & 62.99 \\
\hline $130^{\circ}$ & - & 1.76 & + & 57.06 & + & 58.82 & + & 60.58 & + & 62.34 \\
\hline $140^{\circ}$ & - & 4.22 & + & 49.67 & + & 53.89 & + & 58.11 & + & 62.33 \\
\hline $150^{\circ}$ & - & 7.79 & + & 39.50 & + & 47.29 & + & 5508 & + & 62.87 \\
\hline 160 & - & 11.62 & + & 27.28 & + & 3890 & + & 50.52 & + & 61.14 \\
\hline 170 & - & 14.82 & + & 13.89 & + & 28.71 & + & 42.53 & + & 58.35 \\
\hline $180^{\circ}$ & - & 16.- & + & $0 .-$ & + & $16 .-$ & + & $32 .-$ & + & 48.- \\
\hline
\end{tabular}

Nella fig. 2 sono riprodotte le curve corrispondenti ai valori della tavola per $c=0,2,3$ ossia per $\eta$ uguale a zero, 0,245 (massimo compatibile colla disuguaglianza (35) e 0,368 (oltrepassante tale massimo). 
RAPPRESENTAZIONE ARMONICA DEL CICLO ECC. 257

La ispezione delle prime due di tali curve rende manifesto come esse abbiano un andamento molto simile a quello della

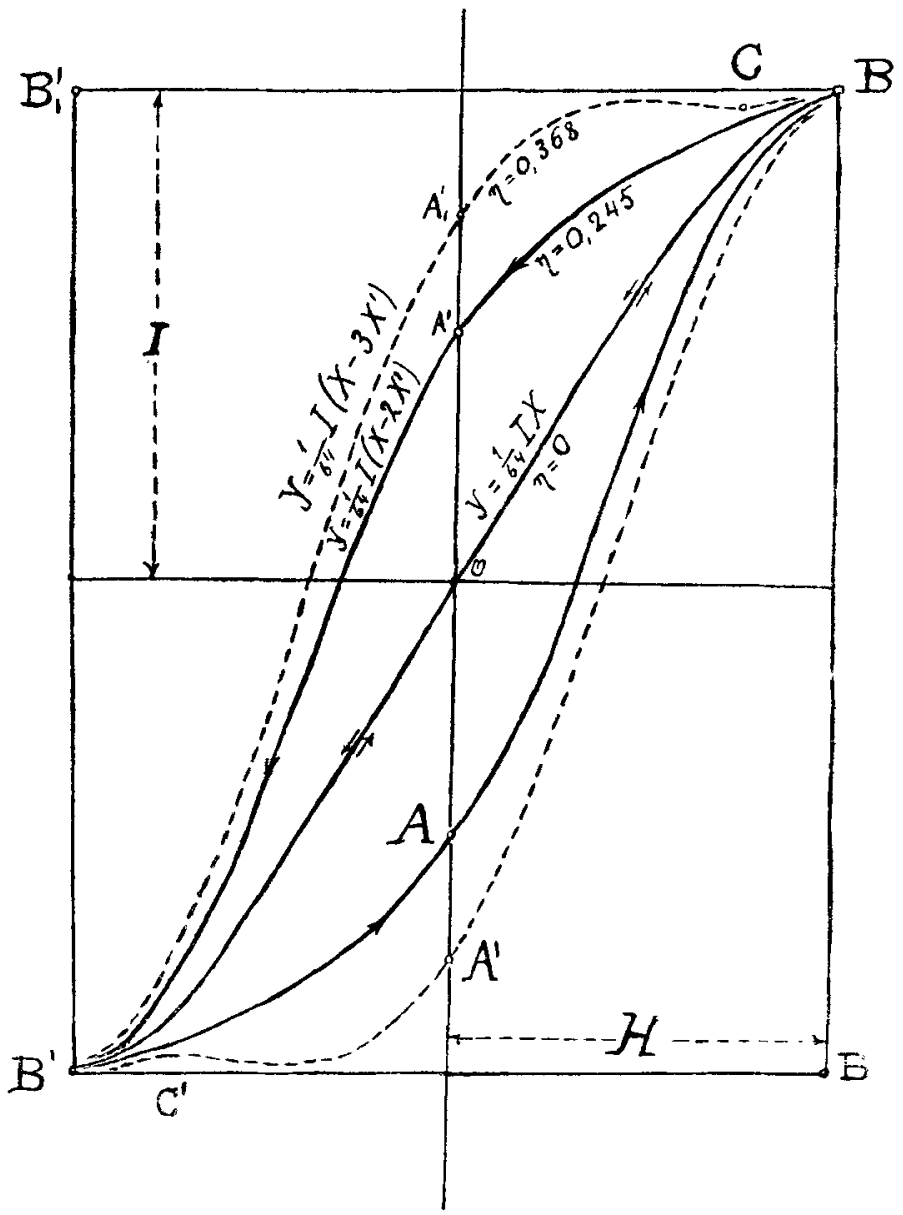

Fig. 2.

$$
\begin{aligned}
& x={ }_{\mathrm{H}} \operatorname{sen} \alpha \\
& v={ }_{64}^{1}\left(\mathrm{X}-8,15 \eta \mathbf{X}^{\prime}\right) \\
& X=70 \operatorname{sen} x+5 \operatorname{sen} 3 x-\operatorname{sen} 5 x \\
& \mathbf{X}=10 \cos z+5 \cos 3 x+\cos 5 \% \\
& \eta={ }_{4 \mathrm{HI}}^{\mathrm{W}}=\frac{\text { area ciclo }}{\text { area rett. circ. }}
\end{aligned}
$$

magnetizzazione ciclica dei materiali ferromagnetici e riproducano in ogni modo realmente le particolarità che abbiamo 
dette caratteristiche del fenomeno: ugualmente si avrebbe per tutte le curve intermedie. La terza invece, cui compete un valore di $\eta$ superiore al massimo indicato dalla disuguaglianza (35), vi si scosta notevolmente, come il calcolo faceva prevedere, specialmente per il fatto che presenta un maggior numero di punti di inflessione.

Certo però che anche le prime due di tali curve, se si accostano molto a quelle realmente seguite dal ferro stirato '), dall'acciaio ecc., corrispondono meno bene per il ferro dolce. La differenza consiste nell' indicare pel magnetismo residuo (38) una frazione della magnetizzazione massima minore di quella che di fatto col ferro dolce non si abbia. Questo inconveniente però non solo sussiste anche nella rappresentazione elittica, ma in questa è più grave. Infatti, dalla (3) si ricava che, per un certo valore di $\eta=\frac{W}{4 \mathrm{HI}^{\prime}}$ con la rappresentazione elittica il magnetismo residuo indicato è di:

$$
\mathrm{I}_{r}=\mathrm{I} \operatorname{sen} \varphi==\frac{4}{\pi} \eta \mathrm{I}=1,27 \eta \mathrm{I}
$$

mentre con la rappresentazione armonica qui proposta $\dot{e} \mathrm{di}$ :

$$
\mathrm{I}_{r}=\frac{32}{5 \pi} \eta \mathrm{I}=1,6 \cdot \frac{4}{\pi} \eta \mathrm{I}=2,04 \eta \mathrm{I} \text {. }
$$

Volendo ottenere maggiore fedeltà nella rappresentazione occorrerebbe far coincidere il magnetismo residuo indicato con quello sperimentale, per il che basterebbe aggiungere alla (9) un termine contenente $u_{7} \cos 7 \alpha$ e porre oltre alle condizioni già esposte anche l'altra:

$$
u_{1}+u_{3}+u_{5}+u_{7}=\mathrm{I}_{r}
$$

dore $I_{r}$ si intenda determinato con esperienze preliminari di magnetizzazione statica. $\dot{E}$ evidente che la introduzione di questo nuovo termine richiederebbe di rifare l'analisi qui compiuta relativamente alle proprietà $(c)$ e $(d)$ e darebbe logo a maggiore complicazione nelle formule finali.

1) EwINa: Magnetic induction in Iron and other Metals. Third edition, pag. 81, 122. 


\section{Osservazioni.}

La rappresentazione data dalla (40), o dalle (42), (43) c (44) che da essa derivano, richiede, come s'è visto:

1.0 che sia trascurabile $\frac{s}{\mathrm{I}}$;

$2 .^{\circ}$ - che il campo applicato varii fra limiti abbastanza eleratı per poter supporre che la curva di isteresi ammetta agli estremi delle cuspidi e non dei punti angolosi;

$3^{\circ}$.. che l'area del ciclo sia inferiore ad una frazione determinata dell'area del rettangolo circoscritto.

In quanto alla prima condizione si osserva che, se non fosse verificata, basterebbe modificare secondo le (34) i coefficienti dei seni nella (40) - e quelli che vi corrispondono nelle altre che da questa derivano - perchè la rappresentazione fosse ancora legittima.

La seconda, mentre è verificata in molte applicazioni di laboratorio, non lo è ugualmente bene, in generale, nelle applicazioni industriali. In queste infatti si cimenta il ferro con campi magnetici variabili entro limiti di sole poche unità, campi che convengono per ottentre indazioni variabili fra limiti molto elevati, - ed in tal caso il ciclo presenta agli estremi dei punti angolosi e non delle cuspidi. Per togliere l'inconveniente - riserbandoci di trattare in altra occasione più a fondo tale argomento - basterà sostituire al ciclo reale uno fittizio di uguale area ed avente le caspidi in modo che il coefficiente angolare della tangonte in queste sia la media aritmetica di quelli delle tangenti ai due rami della curva in ciascun punto angoloso. Con ciò non verranno abbandonati $i$ vantaggi che colla rappresentagicne proposta abbiawo fatto vedere esser stati raggiunti.

La terza infine, ciò̀ la limituzione imposta per il rapporto $\eta$ fra l'area del ciclo di isturesi e quella del rettangolo circoscritto, non è tale da esclutwe l'applicaziono della rappresentazione qui proposta all: ingnetizzazione nelle condizioni di funzionamento degli ancechi industriali. A tale proposito possiamo ricordare i dati sperimentali riportati dal 
prof. Lombardi ") circa le "perdite nel ferro » in un trasformatore della casa Ganz mantenuto col secondario aperto e cogli estremi del primario ad una tensione alternata variabile da 100 - a 1100 volt. con 40 periodi per 1". Tenuto conto che la energia dissipata può essere espressa - nella ipotesi sinusoidale del sampo e della induzione ritardata su quello - da $\mathrm{W}=\pi \mathrm{HI} \operatorname{sen} \varphi$, viene che: $\eta=\frac{\mathrm{W}}{4 \mathrm{HI}}=\frac{1}{4} \pi \operatorname{sen} \varphi$, per cui, introducendo per $\varphi=2 \pi n t$ i valori sperimentali suddetti, si trova che $\eta$ varia da 0,270 a 0,222 . Se si riflet te che nelle " perdite nel ferro "sono conglobate, con quelle dovute alla isteresi, anche quelle dipendenti dalle correnti di Foucault - mentre la rappresentazione proposta nella presente nota $\dot{\theta}$ relativa alla isteresi pura, poichè solo quando si prescinda dalle correnti di Foucault i massimi del campo e della magnetizzazione coincidono - si comprende come facilmente si deỏba scendere con $\eta$ sempre al di sotto del valore 0,245 assegnato come massimo per la validità della rappresentazione.

Meno bene soddisfano i dati riportati dallo stesso antore per un trasformatore della casa Oerlikon, ma anche in questo caso, sottraendo le perdite per correnti di Foucault, probabilmente si scende con $\eta$ sotto il limite necessario.

\section{Conclusione.}

Partendo da proprietà sperimentali caratteristiche dei cicli simmetrici di isteresi magnesicd, e supposto il campo magnetico variabile sinnsoidalmente, siamo giunti ad una rappresentazione analitica della magnetizzazione per mezzo di un termine sinusoidale e dei due armonici dispari successivi. L'ampiezza di ciascuno dei tre termini è proporzionale alla intensità di magnetizzazione mentre il valore rispettivo di ciascuno e lo sfasamento rispetto al campo dipendono esclusivamente dal rapporto fra l'area abbracciata dal ciclo e quella del rettangolo circoscritto.

La funzione ottenuta richiede per la sua validità che il ciclo cui si riferisce sia abbastanza esteso da non avere, sen-

1) Lomrardi: Lezioni di Elettrutecnica. Vol. II, pag. 47. 
RAPPRESLNTAZIONE ARMONICA DEL CICLO ECC. 261

sibilmente, punti angolosi ai suoi estremi ma delle cuspidi e da racchiudere un'area che non sia una frazione troppo grande di quella del rettangolo circoscritto. In quelle applicazioni nelle quali la prima di tali condizioni non sia verificata si sostituirà al ciclo reale di isteresi un ciclo fittizio opportunamente scelto.

Essa funzione, per quanto non sempre riproduca tutte le particolarita del fenomeno, - in particolare non indicando pel magnetismo residuo il suo valore sperimentale per tutti i materiali - segue il fenomeno stesso più da ricino di quanto non facciano altre formule proposte precedentemente ed, in particolare, più da vicino che non l'elisse usata ordinariamente in elettrotecnica. La sua forma analitica periodica si presta quanto quella elittica - salvo la inevitabile maggiore complicazione - ad essere introdotta senz'altro nello studio delle correnti alternate.

Alla funzione abbiamo date varie forme da impiegarsi nei differenti casi.

Osserviamo che la rappresentazione ottenuta, pur avendo di mira la isteresi magnetica, potrà servire anche in altri casi di isteresi, purchè simmetrica. 